\title{
Ionization Suppression and Recovery in Direct Biofluid Analysis using Paper Spray Mass Spectrometry
}

\author{
Carolina Vega, Corina Spence, Chengsen Zhang, Brandon J. Bills, and Nicholas E. Manicke \\ Department of Chemistry and Chemical Biology, Indiana University-Purdue University Indianapolis, Indianapolis, \\ Indiana 46202, United States
}

Paper spray mass spectrometry is a method for the direct analysis of biofluids samples in which extraction of analytes from dried biofluids spots and electrospray ionization occur from the paper on which the dried sample is stored. We examined matrix effects in the analysis of small molecule drugs from urine, plasma, and whole blood. The general method was to spike stable isotope labeled analogs of each analyte into the spray solvent, while the analyte itself was in the dried biofluid. Intensity of the labeled analog is proportional to ionization efficiency, whereas the ratio of the analyte intensity to the labeled analog in the spray solvent is proportional to recovery. Ion suppression and recovery were found to be compound and matrix dependent. Highest levels of ion suppression were obtained for poor ionizers (e.g. analytes lacking basic aliphatic amine groups) in urine and approached -90\%. Ion suppression was much lower or even absent for good ionizers (analytes with aliphatic amines) in dried blood spots. Recovery was generally highest in urine and lowest in blood. We also examined the effect of two experimental parameters on ion suppression and recovery: the spray solvent and the sample position (how far away from the paper tip the dried sample was spotted). Finally, the change in ion suppression and analyte elution as a function of time was examined by carrying out a paper spray analysis of dried plasma spots for 5 minutes by continually replenishing the spray solvent.

This is the author's manuscript of the article published in final edited form as:

Vega, C., Spence, C., Zhang, C., Bills, B. J., \& Manicke, N. E. (2016). Ionization Suppression and Recovery in Direct Biofluid Analysis Using Paper Spray Mass Spectrometry. Journal of The American Society for Mass Spectrometry, 27(4), 726-734. https://doi.org/10.1007/s13361-015-1322-8 


\section{Introduction}

There is an abiding interest in reducing the complexity of mass spectrometric analyses by reducing or eliminating sample preparation and chromatography. Progress in this field accelerated rapidly with the invention of desorption electrospray ionization (DESI)[1] and direct analysis in real time (DART)[2]. Paper spray is a member of this growing cohort of ambient ionization or direct analysis methods that was conceived because of the growing interest in storing and transporting blood samples as dried spots. One of the major applications of paper spray mass spectrometry has been screening and quantitative analysis of drugs and other small molecules from biofluids such as blood, urine and plasma[3-15]. Other applications include analysis of agrochemicals and contaminants in food[16-18], tissue analysis[19], surface swabbing[20,21], profiling of algae[22] and bacteria[23, 24], cell culture monitoring[25], and herbicides in water and crop extracts[26]. In addition to applications development, methodological advancements have been reported for paper spray MS such as improving selectivity using differential mobility spectrometry prior to MS detection[27], improving sensitivity using solid phase extraction[28], the use of alternative porous substrates[9, 29-31], and using paper spray as an ionization source for microfluidics chips[32, 33]. Additionally, solid phase microextraction (SPME)-type approaches have been reported in which the SPME material itself served as the spray substrate[34, 35].

Paper spray is performed by depositing a few microliters of the sample, such as blood or urine, onto paper that has been cut to a sharp point (often triangular shaped though not necessarily). A solvent is applied to the paper, where it wicks through the paper by capillary action. As the solvent wicks through the sample, soluble analytes and matrix components are extracted into the spray solvent. After the solvent has wicked to the tip of the paper (typically within seconds), electrospray ionization is induced at the sharp tip of the wet paper via the application of a high voltage. Analytes are typically detected immediately, although separation effects have been reported in some cases[36, 37]. Analyte signal can last for several minutes, allowing multiple analytes to be analyzed by $\mathrm{MS}^{\mathrm{n}}$. Quantitation can be done by integration of the analyte signal for some defined length of time followed by normalizing with the corresponding area under the curve for the internal standard.

Matrix effects are known to occur in paper spray mass spectrometry when analyzing dried biofluids[4, 14, 28]. MS signal intensities obtained from the analysis of dried urine, blood, or plasma are almost always lower compared to analyzing an identical quantity of analyte without the presence of biofluids matrices. The presence of matrix effects does not preclude quantitative analysis provided that matrix matched calibrators and stable isotope labeled (SIL) internal standards are used, as has been demonstrated in the literature numerous times. Also, despite the presence of matrix effects, surprisingly good detection limits in the single digit or sub-ng/mL range are widely reported in the literature for direct analysis of dried blood spots. Nevertheless, minimizing or eliminating matrix effects in paper spray MS would be beneficial because detection limits would be lowered and the need for matrix matched calibrations and SIL internal standards could be eliminated. To take rational steps to minimize matrix effects, a more rigorous understanding of the causes and the experimental parameters that impact matrix effects is first needed.

In this study, we examined the contribution of ion suppression and recovery to matrix effects in the analysis of small molecule drugs in various biofluids. We also examined two experimental parameters that impact the magnitude of matrix effects: extraction/spray solvent and the position of the dried biofluids sample on the paper spray substrate. Finally, we studied 
the change in ion suppression and analyte elution as a function of time during a 5 minute long paper spray analysis.

\section{$\underline{\text { Methods }}$}

Materials. Methanol, acetonitrile, and acetic acid (HPLC grade) were purchased from Fisher Scientific (Hampton, New Hampshire). Most drugs and stable isotopically labeled analogs were purchased from Cerilliant (Reston, VA, USA) as $1 \mathrm{mg} / \mathrm{mL}$ or $100 \mu \mathrm{g} / \mathrm{mL}$ stock solutions and stored at $-20{ }^{\circ} \mathrm{C}$. Exceptions were sulfamethazine (Sigma, St Louis, MO, USA), ${ }^{2}[\mathrm{H}]_{4-}$ sulfamethazine and ${ }^{2}[\mathrm{H}]_{7}$-atenolol (CDN Isotopes, Pointe-Claire, Quebec, Canada). Whatman grade 31ET-Chr paper was purchased from Whatman (Piscataway, NJ, USA). Human blood and urine were obtained from a single donor. Blood was collected into $\mathrm{K}_{2}$ EDTA blood collection tubes. Plasma was isolated from freshly collected blood by centrifugation.

Paper spray mass spectrometry. 31ET chromatography paper was used for both the spray substrates and the sample discs. All experiments were carried out on an LTQ-XL mass spectrometer (Thermo Scientific, San Jose, CA) in operated in MS/MS mode unless noted otherwise. The primary fragment ion monitored for each analyte and the collision energy used for each analyte is shown in Supplementary Table 2. The extraction/spray solvent was 95-5-0.01 methanol-water-acetic acid unless noted otherwise. Taylor cone formation and stability were observed in real time using a Pulnix TM-200 camera equipped with a Precise Eye adapter tube and lens attachment (1-61448 and 1-61449, Navitar, Rochester NY).

Paper spray ionization was carried out using two experimental set-ups. Cartridge A (Figure 1A) consisted of a Delrin plastic holder with a slot milled into the side to hold the spray substrate, which was an irregular pentagonal shaped piece of 31ET chromatography paper, $5 \mathrm{~mm}$ wide at the base and $10 \mathrm{~mm}$ in length. A hole was drilled perpendicular to the top surface of the spray substrate to hold one or more paper discs with a thickness of $\sim 0.5 \mathrm{~mm}$. The sample was spotted on one of these discs. The position of the dried sample within a stack of paper discs was changed to easily manipulate the sample position (a vertical distance of 0-1.5 mm away from the pentagonal shaped paper) while keeping other experimental variables constant (like amount of paper and solvent volume). Cartridge B consisted simply of a piece of delrin plastic with a trench milled out to hold a $25 \mathrm{~mm}$ long, $6.3 \mathrm{~mm}$ wide strip of paper cut to a sharp point (Figure 1B). Samples were spotted at $6.3,12.5$, or $23.5 \mathrm{~mm}$ away from the tip of the spray substrate. Cartridges were made on a bench top milling machine (Sherline, Vista, CA).

For the cartridge $\mathrm{A}$, the sample volume was $3 \mu \mathrm{L}$ and the volume of spray solvent varied from $30 \mu \mathrm{L}$ to $45 \mu \mathrm{L}$ depending on the number of paper discs above the paper spray substrate. The data acquisition time was typically 45 seconds. For the experiments in which data acquisition was extended to 5 minutes, $10 \mu \mathrm{L}$ of additional spray solvent was added to the cartridge every minute. For cartridge B, the sample volume was $6 \mu \mathrm{L}$ and the spray solvent volume was $120 \mu \mathrm{L}$. The entire volume of spray solvent was added slowly at the back-end of the paper, approximately matching the wicking rate of the solvent through the paper, before applying the spray voltage. To perform the analysis, the paper tip was positioned 5 millimeters away from the atmospheric pressure inlet of the mass spectrometer, and the extraction/spray solvent was applied just prior to application of $4.5 \mathrm{kV}$ to the paper using the built-in high voltage power supply on the MS. The cartridges were positioned in front of the mass spectrometer using a 3 
axis micrometer affixed to a stage which was attached to the mass spectrometer via the same latch system used for the commercial ion source.

Sample preparation. Drug samples were prepared at a concentration of $500 \mathrm{ng} / \mathrm{mL}$ by 1:20 dilution of $10 \mu \mathrm{g} / \mathrm{mL}$ working solutions in 1:1 methanol:water into the biofluid. Working solutions were stored at $-20^{\circ} \mathrm{C}$ and biofluids samples were prepared fresh daily. Samples spotted on paper were allowed to dry at room temperature prior to analysis.

\section{$\underline{\text { Results and Discussion }}$}

Ion suppression and recovery

The mass spectrometric signal obtained from analytes in complex matrices is generally lower compared to analytes in pure solvent. Table 1 shows the signal obtained from paper spray ionization of several drugs in dried urine, plasma, and whole blood relative to the same quantity of analyte dried on the paper without the presence biofluids (hereafter referred to as a "matrix free" sample). The absolute signal intensity varied significantly among the five drugs even without the presence of the biological matrix solution due to their varying ionization efficiencies, recoveries from the matrix free paper, and fragmentation behaviors. When present in dried biofluids, the decrease in analyte signal was both compound and matrix dependent. On average, the analyte signal from dried biofluids was about 10 times lower compared to matrix free samples, ranging from signal drop of a factor of 2 for methadone in urine to a factor of around 25 for several of the drugs in blood and plasma.

The matrix effects arise from a combination of lower analyte ionization efficiency (ion suppression) and lower recovery. To determine the relative importance of these factors, we spiked stable isotopically labeled (SIL) analogs of each analyte into the spray solvent at a concentration of $20 \mathrm{ng} / \mathrm{mL}$. If the recovery $(R)$ is defined to be the amount of analyte present in the detection volume (i.e. the solvent that is sprayed over the duration of the experiment) divided by the total amount of analyte present in the sample and the ionization efficiency $(E)$ is defined to be the amount of ionized analyte divided by the amount of analyte present in the detection volume, then

$$
\begin{aligned}
& A U C_{A} \propto[A] R E \\
& A U C_{L} \propto[L] E
\end{aligned}
$$

where $A U C_{A}$ and $A U C_{L}$ are the areas under the curve for the analyte and SIL analog, respectively, $[A]$ is the concentration of the analyte in the sample, and $[L]$ is the concentration of the SIL analog in the spray solvent. Proportionality rather than equality is indicated because there are other variables (such as total sample amount) which we don't consider but hold equal among all of the experimental groups. Proportionality will also only hold over a certain concentration range, which is compound and matrix dependent. Finally, determination of the absolute values for $R$ and $E$ is not be attempted. Rather, we focus only on the relative change between among experimental conditions. The relative recovery from dried biofluid samples compared to matrix free samples is equal to the ratio of the analyte intensities divided by the ratio of ionization efficiencies: 


$$
\begin{gathered}
\frac{R_{\text {biofluid }}}{R_{\text {neat }}}=\frac{A U C_{A, \text { biofluid }}}{A U C_{A, \text { neat }}} / \frac{E_{\text {biofluid }}}{E_{\text {neat }}} \\
\frac{E_{\text {biofluid }}}{E_{\text {neat }}}=\frac{A U C_{L, \text { biofluid }}}{A U C_{L, \text { neat }}}(4)
\end{gathered}
$$

The ion suppression (as a percent decrease in the ionization efficiency compared to matrix free samples) is equal to

$$
\frac{A U C_{L, \text { biofluid }}-A U C_{L, \text { neat }}}{A U C_{L, \text { neat }}}
$$

The relative recovery and ion suppression for 5 drugs in urine, plasma, and blood are shown in Table 2. For each of the five drugs, ion suppression was highest (i.e. lowest ionization efficiency) in urine samples, followed by plasma, with blood showing the least ion suppression. Conversely, blood exhibited the lowest recovery, followed by plasma, with urine showing the highest recovery. It should be noted that the recovery from matrix free paper is much less than $100 \%$. The absolute recovery is therefore lower than that shown in Table 2.

Concerning ion suppression, there seemed to be a trend related to the $\mathrm{p} K_{\mathrm{a}}$ (conjugate acid) of the weakest base that must be protonated in order to give the analyte a +1 charge. In the case of morphine and methadone, those groups are the relatively basic aliphatic amines with $\mathrm{p} K_{\mathrm{a}}$ over 9. These two analytes showed the lowest ion suppression. Alprazolam, carbamazepine, and benzoylecgonine, on the other hand, all have groups with a $\mathrm{p} K \mathrm{a}$ of 5 or less that must be protonated (heterocyclic amine, amide, and carboxylic acid, respectively). These three analytes showed higher levels of ion suppression. These results indicate that more basic analytes (whose conjugate acids' have higher $\mathrm{p} K_{\mathrm{a}} \mathrm{s}$ ) tend to show lower levels of ion suppression, which is consistent with the expected behavior of electrospray ionization[38].

\section{Effect of extraction/spray solvent on ion suppression and recovery}

We investigated two common paper spray solvents to compare ion suppression and recovery: 90-10-0.01 acetonitrile-water-acetic acid and 95-5-0.01 methanol-water-acetic acid. Comparison of the ion suppression and recovery for the two solvent systems are shown in Table 3 . The results are presented as the relative difference between the acetonitrile based solvent and the methanol based solvent. The acetonitrile based solvent generally showed lower levels of ion suppression (higher ionization efficiency) but also somewhat lower recovery than the methanol solvent. The difference in analyte signal intensity between the two solvents, which is a function of both ion suppression and recovery, was negligible for matrix free samples and dried blood samples. In dried plasma and urine, acetonitrile gave significantly higher analyte signal for the three analytes most affected by ion suppression (alprazolam, carbamazepine, and benzoylecgonine). This is consistent with the results described in the previous section; because acetonitrile showed lower ion suppression, it tended to yield higher analyte signal intensities for matrix/analyte combinations exhibiting the most ion suppression. The data for the absolute intensities are shown in Supplementary Table 1

\section{Effect of plasma sample position on ion suppression and recovery}

We investigated the effect of increasing the distance between the sample and the paper tip (i.e. changing the amount of paper the extract solvent must wick through after interacting with 
the sample). Two experimental setups were used to study the effect of sample position on analyte signal intensity, ion suppression, and recovery as shown in Figure 1

For matrix free samples, the area under the curve (AUC) measured for 45 seconds after applying the spray voltage decreased uniformly as the distance between the sample and the spray tip increased (Supplemental Figure 1, using cartridge B). When spiked into plasma, the AUC measured during the first 45 seconds increased as the sample was moved farther away from the spray tip for some drugs and decreased for others (Figure 2, using cartridge A). The five drugs that showed decreasing MS signal with sample distance all contain aliphatic amines with aqueous $\mathrm{p} K_{\mathrm{a}} \mathrm{s}$ (conjugate acid) in the 8-10 range. The four that showed higher signal either do not have aliphatic amines or, in the case of benzoylecgonine, have an aliphatic amine but also have a carboxylic acid group. The aqueous $\mathrm{p} K_{\mathrm{a}}$ of the heterocyclic amine conjugate acids and the carboxylic acid group of benzoylecgonine are in the 2-5 range, many orders of magnitude less basic than the aliphatic amines in the other group of drugs (atenolol, imipramine, methadone, morphine, and sunitinib). Similar trends were also observed using cartridge B, in which the distance scale is bigger than that of cartridge A (Supplemental Figure 2).

To investigate the factors that cause MS signal intensity to change as a function of sample position, we again spiked SIL analogs of the drug into the spray solvent. The AUC during the first 45 seconds for the unlabeled drug (spiked into the plasma sample) and the SIL analog (spiked into the extraction/spray solvent) obtained for various sample positions are shown in Figure 3 for alprazolam and morphine using cartridge A. Data from a similar experiment using cartridge B is shown in Supplementary Figure 3.

The change in the analyte and SIL analog intensities, as well as the ratio of the intensities, as a function of sample position ( $\mathrm{x}$ ) can be expressed as follows by taking the partial derivative of equations 1 and 2 with respect to the sample position:

$$
\begin{gathered}
\frac{\partial\left(A U C_{A}\right)}{\partial x} \propto[A]\left(E \frac{\partial R}{\partial x}+R \frac{\partial E}{\partial x}\right) \\
\frac{\partial\left(A U C_{L}\right)}{\partial x} \propto[L] \frac{\partial E}{\partial x} \\
\frac{\partial\left(\frac{A U C_{A}}{A U C_{L}}\right)}{\partial x}=\frac{[A]}{[L]} \frac{\partial R}{\partial x}
\end{gathered}
$$

Over the distances we examined for the 9 analytes in Figure $2, \frac{\partial R}{\partial x}$ was always negative and $\frac{\partial E}{\partial x}$ was always positive for dried plasma samples. In other words, recovery (as defined above) decreased when the sample was farther from the spray tip while ionization efficiency increased. Moving the sample farther away from the tip therefore decreased or increased the analyte signal depending on the magnitudes of $\frac{\partial R}{\partial x}, \frac{\partial E}{\partial x}, R(x)$, and $E(x)$. As shown in Figure 3 , alprazolam signal improved as the sample was moved farther away from the spray tip because alprazolam showed a significant decrease in ion suppression. Morphine, on the other hand, showed lower signal as the sample was moved farther away because it is a relatively basic compound and showed only modest the improvement in ionization efficiency when moving the dried plasma sample farther away. Sulfamethazine, benzoylecgonine, and carbamazepine showed similar trends to alprazolam. Atenolol, imipramine, methadone, and sunitinib showed similar trends to morphine (data not shown). 
The increase in analyte ionization efficiency as the sample is moved farther away from the tip is presumably caused by a decrease in ion suppression, possibly due to some components of the plasma matrix being more strongly retained by the paper than the drug targets. To test this hypothesis, the drug analytes were spiked into the spray solvent at $20 \mathrm{ng} / \mathrm{mL}$. The signal intensity of the drugs was monitored as a function of time with a blank dried plasma spot in various positions. Drug spiked spray solvent was added to the cartridge at regular intervals to maintain a stable spray. The chromatograms obtained for alprazolam using cartridge A with four paper discs in the cartridge are shown in Figure 4. When no plasma was present, the signal of alprazolam was essentially stable for the duration of the experiment (5 minutes). When blank dried plasma was added to the first punch, the MS signal for alprazolam decayed noticeably within 30 seconds. Moreover, the initial intensity was nearly a factor of 10 lower than when no plasma was present. As the plasma was moved farther from the spray substrate by changing which punch contained the dried plasma, the alprazolam signal decay was delayed and initial signal intensity increased until it was approximately the same as when no plasma was present.

The decrease in signal intensity appears to be associated with an increase in signal for some components from the plasma. Figure 5 shows the extracted ion chromatograms for sulfamethazine and atenolol (dissolved in the spray solvent) as well as two lipids detected from the plasma matrix (the phospholipid 34:2 glycerophosphocholine and the metabolite choline). The sulfamethazine signal falls off rapidly as the signal from matrix components increase. The ionization efficiency of atenolol, having an aliphatic amine, is not as strongly suppressed as alprazolam and sulfamethazine but still decreases as the signal for the matrix components increase.

Ion suppression and recovery versus time

In typical paper spray analyses, the high voltage is applied for some defined length of time (often 30-60 seconds) and the analysis is halted by turning the voltage off. Analyte signal can be maintained for longer times, although solvent must be replenished in order to maintain a stable electrospray. As a function of time, both the instantaneous analyte concentration in the spray solvent and the ionization efficiency may change. Moreover, there can also be changes in the physical parameters of the electrospray including changes in droplet size distribution and flow rate[39]. These changes are thought to occur due to solvent depletion when the spray solvent is not replenished during analysis. In order to study changes in ion suppression and analyte elution as a function of time, we analyzed analyte spiked dried plasma samples using spray solvent spiked with SIL analogs of each analytes over the course of 5 minutes. The solvent was replenished at regular intervals in order to keep the paper saturated and maintain a stable electrospray and a consistent spray mode. A stable Taylor cone was observed in these experiments and the spray current remained constant at about $0.3 \mu \mathrm{A}$. The change in analyte intensity (in the dried plasma sample) and SIL analog intensity (dissolved in spray solvent) as a function of time can be expressed as:

$$
\begin{gathered}
\frac{\partial I_{A}}{\partial t} \propto[A]\left(E \frac{\partial R^{\prime}}{\partial t}+R^{\prime} \frac{\partial E}{\partial t}\right) \\
\frac{\partial I_{L}}{\partial t} \propto[L] \frac{\partial E}{\partial t}
\end{gathered}
$$




$$
\frac{\partial\left(\frac{I_{A}}{I_{L}}\right)}{\partial t}=\frac{[A]}{[L]} \frac{\partial R^{\prime}}{\partial t}
$$

Where $I_{A}$ and $I_{L}$ are the intensities obtained for the analyte and the SIL analog, respectively. $R^{\prime}$ is the amount of analyte in the solvent volume detected during a single MS scan event divided by the total amount of analyte in the sample. Relating this to the original definition of $R$ :

$$
R=\sum_{t=0}^{n} R^{\prime}(t)
$$

where $\mathrm{n}$ is the number of MS scan events during the paper spray analysis and the number of scan events that are summed to determine the AUC.

$I_{A}, I_{L}$, and $I_{A} / I_{L}$ as a function of time for atenolol and sulfamethazine are shown in Figure 6. This experiment was performed using cartridge A with four paper discs positioned above the spray substrate. The dried plasma sample was on the third punch from the spray substrate, corresponding to the distance of $1.0 \mathrm{~mm}$ using cartridge A. For both drugs, the ionization efficiency, as indicated by $I_{L}$, started at a maximum value at the beginning of the analysis and then decreased until about 3 minutes. From 3 to 5 minutes (when the experiment was halted), the ionization efficiency was constant. The decrease in ionization efficiency was much more dramatic for sulfamethazine than for atenolol. The ionization efficiency of sulfamethazine decreased by about a factor of 50, compared to only a factor of 2 for atenolol. Several more compounds were also studied (data not shown). Propranolol, imipramine, sunitinib, amitriptyline, and morphine showed similar trends to atenolol. Carbamazepine, temazepam, and oxazepam showed similar trends to sulfamethazine. This is consistent with the earlier observation that more basic compounds (typically those containing aliphatic amines) are less affected by ion suppression than less basic compounds (such as those containing heterocyclic amines or a carboxylic acid group).

For both sulfamethazine and atenolol, $R^{\prime}$ (as measured by the ratio of $I_{A}$ to $I_{L}$ ) started at a minimum value and increased over the first two minutes of the analysis (Figure 6). From 2 to 5 minutes, $R^{\prime}$ remained constant. For sulfamethazine, $I_{A} / I_{L}$ was unstable after two minutes because $I_{A}$ and $I_{L}$ were both very low due to ion suppression. $R^{\prime}$ presumably must decrease again due to depletion of the analyte, but we did not observe this for any of the analytes studied over the duration of this experiment ( 5 minutes).

\section{Conclusion}

Ion suppression and recovery was studied for several drugs in different biological fluids. Ion suppression was found to vary depending on the matrix and the intrinsic properties of the analyte, particularly its basicity. Ion suppression was generally highest in urine and lowest in blood. Recovery, on the other hand, was lowest in blood and highest in urine. Two experimental parameters, spray solvent and sample position, were studied to determine their impact on ion suppression and recovery. An acetonitrile based solvent system was found to give lower ion suppression but often lower recovery as well. Sample position (i.e. moving the dried biofluids sample farther or closer to the spray tip) was also found to impact ion suppression and recovery. Analytes that are relatively strongly affected by ion suppression tended to show improvement as the sample is moved farther away from the tip of the paper where ionization takes place. 


\section{Acknowledgements}

This project was supported by Award No. 2014-R2-CX-K007 awarded by the National Institute of Justice, Office of Justice Programs, U.S. Department of Justice. The opinions, findings, and conclusions or recommendations expressed in this publication are those of the author(s) and do not necessarily reflect those of the Department of Justice.

C.V. acknowledges support from the Brazil Scientific Mobility Program. 
Table 1. Matrix effects in paper spray mass spectrometry. The MS/MS signal intensity obtained during analysis of 3 different biofluids was compared to an equal quantity of analyte dried on matrix free paper $(1.5 \mathrm{ng})$

\begin{tabular}{|c|c|c|c|c|}
\hline & \multicolumn{2}{|c|}{ analyte signal relative to matrix free paper } & absolute signa* - \\
matrix free paper
\end{tabular}

*the area under the curve (AUC) for the primary fragment ion during the first 45 seconds of analysis 
Table 2. Ion suppression and recovery in 3 different dried biofluids. Analytes were analyzed simultaneously at $1.5 \mathrm{ng}$ each. Ion suppression (shown as a percent decrease) and percent recovery were calculated relative to the same analyte quantity dried on matrix free paper.

\begin{tabular}{|c|c|c|c|c|c|c|}
\hline & \multicolumn{3}{|c|}{$\begin{array}{c}\text { ion suppression relative to } \\
\text { matrix free paper }\end{array}$} & \multicolumn{3}{|c|}{ recovery relative to matrix free } \\
paper \\
\hline analyte & urine & plasma & blood & urine & plasma & blood \\
\hline alprazolam & $-90 \%$ & $-80 \%$ & $-40 \%$ & $110 \%$ & $22 \%$ & $9 \%$ \\
\hline benzoylecgonine & $-89 \%$ & $-77 \%$ & $-43 \%$ & $69 \%$ & $17 \%$ & $7 \%$ \\
\hline carbamazepine & $-93 \%$ & $-88 \%$ & $-48 \%$ & $73 \%$ & $21 \%$ & $8 \%$ \\
\hline methadone & $-30 \%$ & $-13 \%$ & $1 \%$ & $79 \%$ & $21 \%$ & $6 \%$ \\
\hline morphine & $-66 \%$ & $-52 \%$ & $-16 \%$ & $44 \%$ & $42 \%$ & $6 \%$ \\
\hline
\end{tabular}


Table 3. Comparison of ionization efficiency and recovery for 2 solvent systems. Solvent A is 90-10-0.01 acetonitrile-water-acetic acid. Solvent B is 95-5-0.01 methanol-water-acetic acid.

\begin{tabular}{|c|c|c|c|c|c|c|c|c|}
\hline \multirow[b]{2}{*}{ analyte } & \multicolumn{4}{|c|}{$\begin{array}{l}\text { ratio of ionization efficiency, solvent } \\
\text { A to solvent B }\end{array}$} & \multicolumn{4}{|c|}{$\begin{array}{l}\text { ratio of recovery, solvent A to solvent } \\
\text { B }\end{array}$} \\
\hline & $\begin{array}{c}\text { matrix } \\
\text { free }\end{array}$ & urine & plasma & blood & $\begin{array}{l}\text { matrix } \\
\text { free }\end{array}$ & urine & plasma & blood \\
\hline alprazolam & 1.6 & 6 & 4 & 1.9 & 0.4 & 0.9 & 1.5 & 0.5 \\
\hline benzoylecgonine & 1.3 & 3 & 5 & 2.0 & 0.4 & 0.8 & 2.0 & 0.7 \\
\hline carbamazepine & 2.1 & 6 & 9 & 2.3 & 0.4 & 0.7 & 2.0 & 0.6 \\
\hline methadone & 0.9 & 0.8 & 1.1 & 0.9 & 0.4 & 0.8 & 1.2 & 0.4 \\
\hline morphine & 1.1 & 1.3 & 2.2 & 1.3 & 1.0 & 0.9 & 3 & 0.7 \\
\hline
\end{tabular}



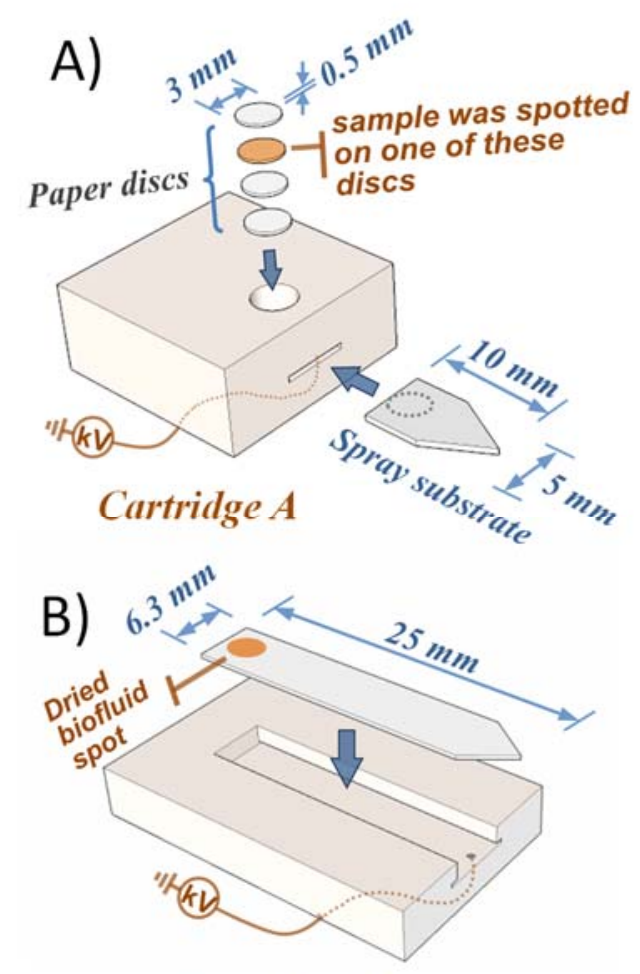

Cartridge $B$

Figure 1. Two approaches were used to perform paper spray in this study. Cartridge A held paper discs in place above the spray substrate. In this figure, a dried plasma sample is shown on the third punch, with a total of 4 punches. Cartridge B consisted simply of a piece of delrin plastic with a trench milled out to hold a $25 \mathrm{~mm}$ long strip of paper cut to a point. 


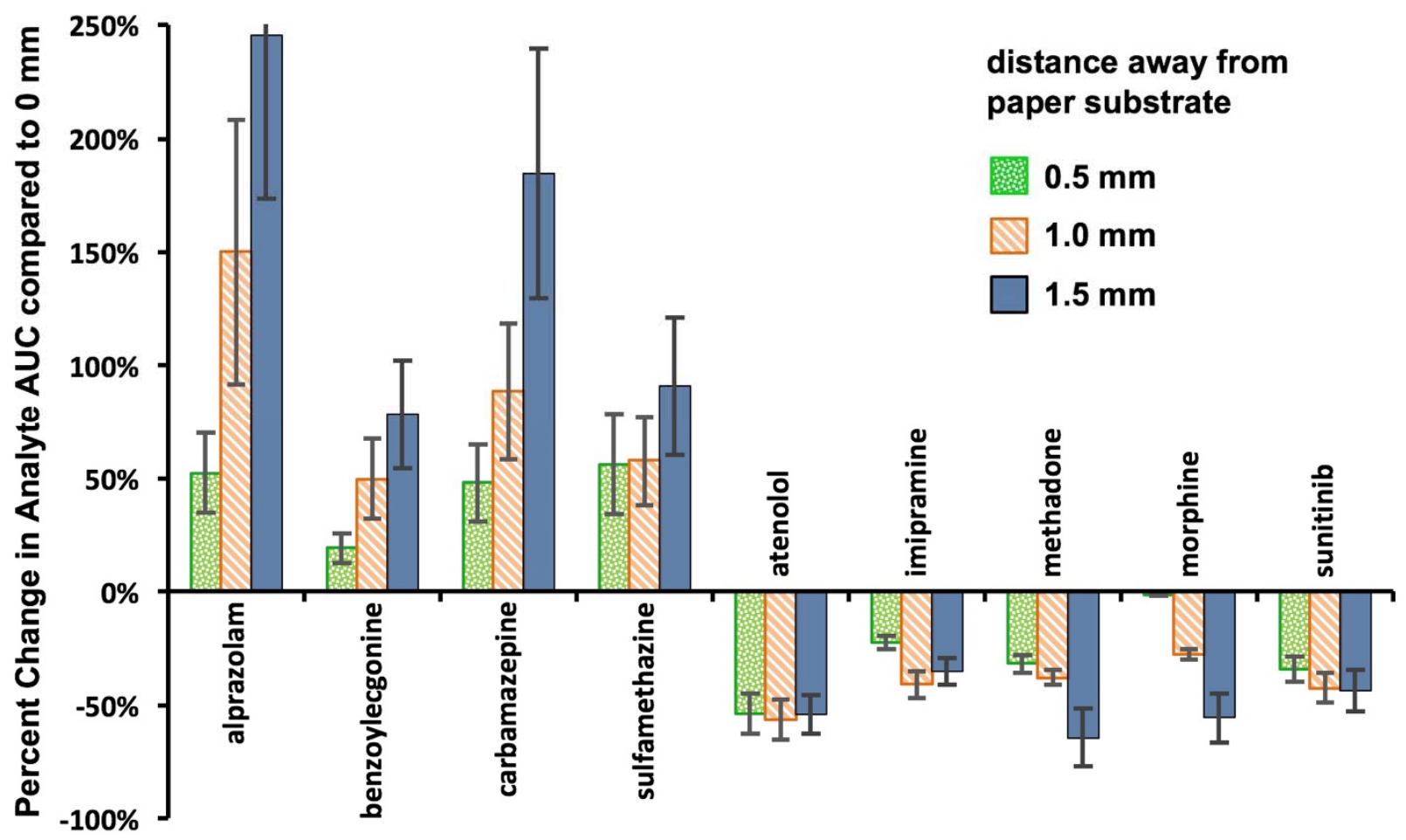

Figure 2. Change in analyte AUC during the first 45 seconds of analysis with sample position. Experiments were done using cartridge A with four paper discs positioned above the paper spray substrate. The position of the dried plasma sample, spiked with $500 \mathrm{ng} / \mathrm{mL}$ of analyte, was varied from the bottom punch ( $0 \mathrm{~mm}$ from spray substrate) to the fourth punch $(1.5 \mathrm{~mm}$ from spray substrate). Percent change was calculated relative to having the dried plasma sample on the bottom punch. 

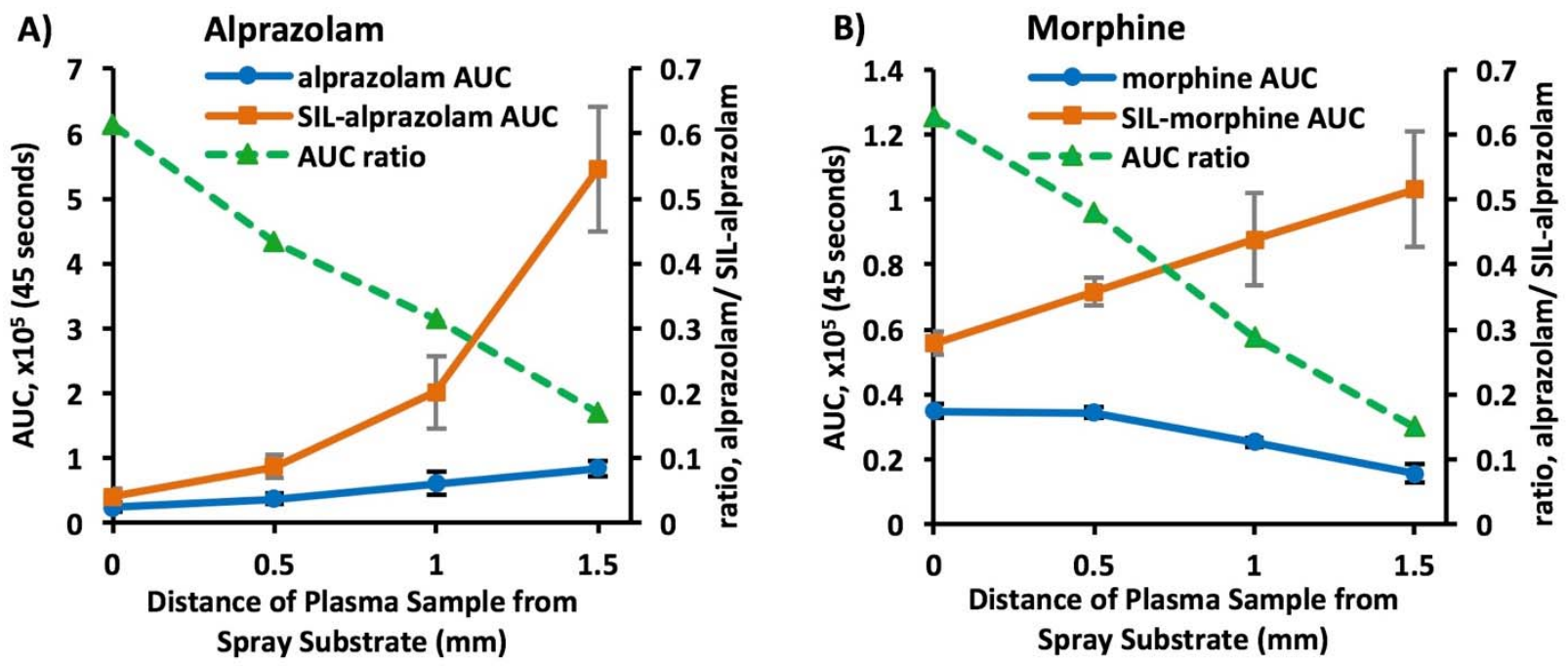

Figure 3. Change in unlabeled analyte AUC (in dried plasma sample), SIL analog AUC (dissolved in spray solvent), and the ratio between the two (plotted on secondary axis) for A) alprazolam, and B) morphine. Experiments were done using cartridge A. The lines linking the data points are included for visual guidance only. 


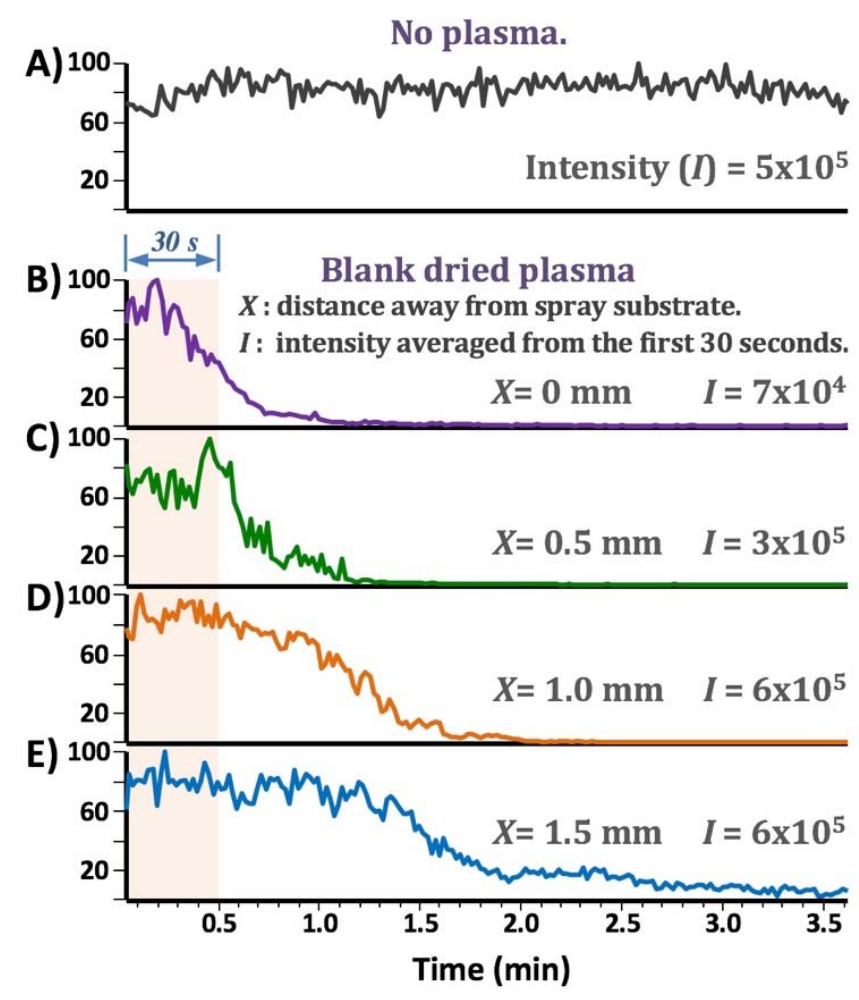

Figure 4. Extracted ion chromatograms for alprazolam (MS/MS, m/z $309 \rightarrow 287$ ) dissolved in the spray solvent and used to extract drug free dried plasma. Cartridge A was used these experiments; four $0.5 \mathrm{~mm}$ thick paper discs were stacked on top of the spray substrate, and the disc containing the drug free dried plasma sample was put at varying positions (from directly on the substrate (B) to the fourth disc (E, $1.5 \mathrm{~mm}$ away from the substrate). Intensity in this figure is the average signal intensity during the first 30 seconds. 


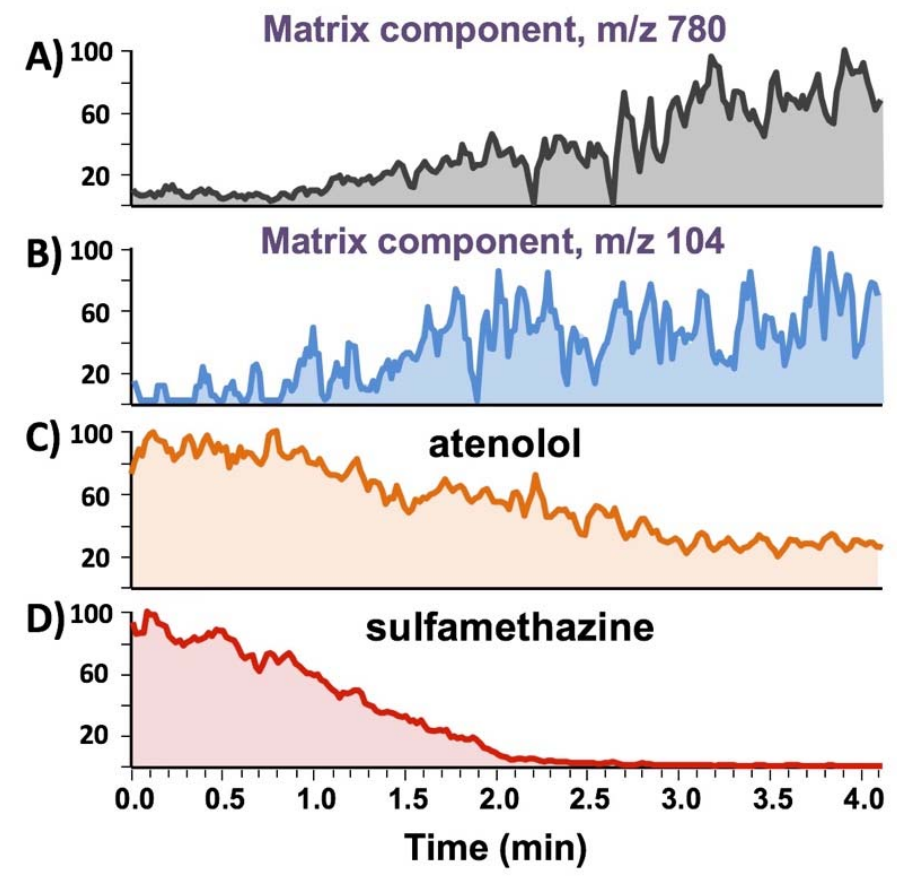

Figure 5. Extracted ion chromatograms for two matrix components from a dried plasma spot (34:2 glycerophospholine sodium adduct, $\mathrm{m} / \mathrm{z} 780$ and choline, $\mathrm{m} / \mathrm{z}$ 104) and two drugs dissolved in the spray solvent at $20 \mathrm{ng} / \mathrm{mL}$. The matrix components were analyzed in full MS mode, while the two drugs were analyzed in MS/MS mode. These experiments were done using cartridge A with 4 paper discs; the blank dried plasma sample was on the $3^{\text {rd }}$ punch from the spray substrate. 

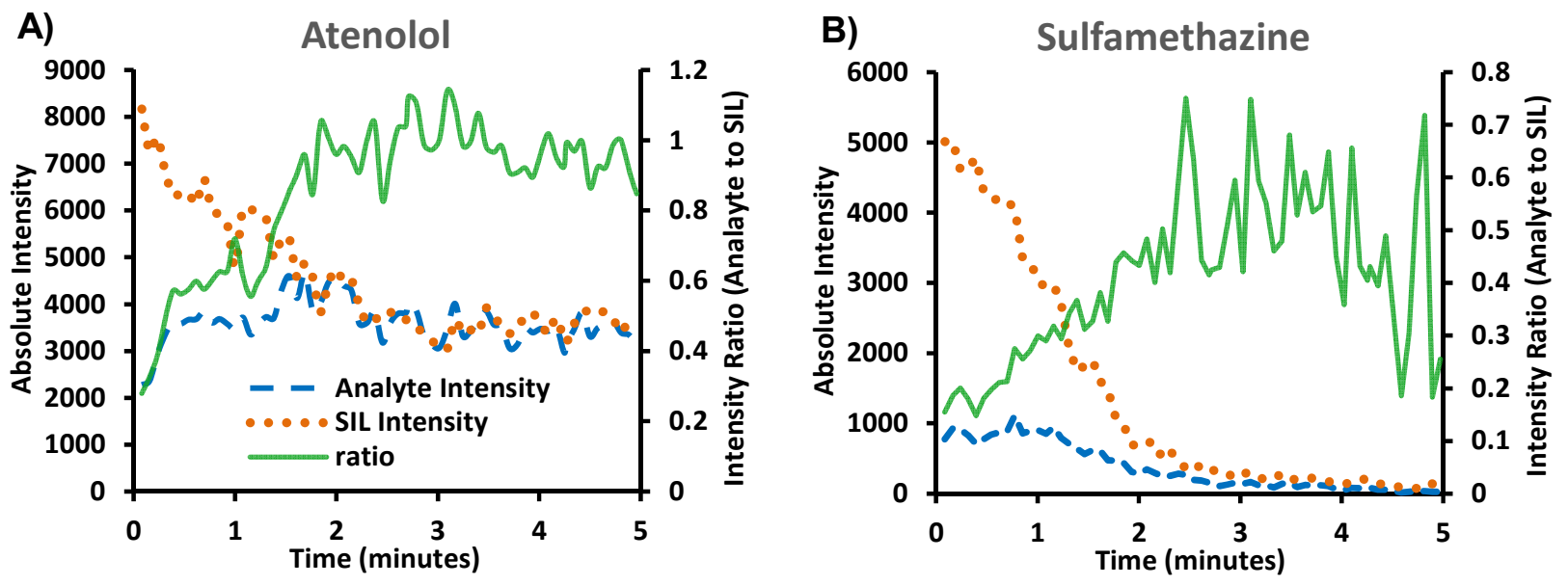

Figure 6. Analyte and SIL analog intensity as a function of time. The ratio of the analyte intensity to SIL intensity is plotted on the secondary axis. Atenolol (A) and sulfamethazine (B) were spiked in the dried plasma sample. ${ }^{2}[\mathrm{H}]_{7}$-atenolol and ${ }^{2}[\mathrm{H}]_{4}$-sulfamethazine were spiked into the spray solvent. Cartridge A was used with 4 paper discs positioned above the spray substrate. The dried plasma sample was on the third punch from the spray substrate. 


\section{References}

1. Takats Z, Wiseman JM, Gologan B, Cooks RG. Mass spectrometry sampling under ambient conditions with desorption electrospray ionization. Science 2004,306, 471-473.

2. Cody RB, Laramee JA, Durst HD. Versatile new ion source for the analysis of materials in open air under ambient conditions. Anal. Chem. 2005,77, 2297-2302.

3. Takyi-Williams J, Dong X, Gong H et al. Application of paper spray-MS in PK studies using sunitinib and benzethonium as model compounds. Bioanalysis 2015,7, 413-423.

4. Manicke NE, Yang Q, Wang H, Oradu S, Ouyang Z, Cooks RG. Assessment of paper spray ionization for quantitation of pharmaceuticals in blood spots. International Journal of Mass Spectrometry 2011,300, 123-129.

5. Manicke NE, Abu-Rabie P, Spooner N, Ouyang Z, Cooks RG. Quantitative Analysis of Therapeutic Drugs in Dried Blood Spot Samples by Paper Spray Mass Spectrometry: An Avenue to Therapeutic Drug Monitoring. Journal of the American Society for Mass Spectrometry 2011,22, 1501-1507.

6. Espy RD, Manicke NE, Ouyang Z, Cooks RG. Rapid analysis of whole blood by paper spray mass spectrometry for point-of-care therapeutic drug monitoring. Analyst 2012,137, 2344-2349.

7. Zhang Z, Xu W, Manicke NE, Cooks RG, Ouyang Z. Silica Coated Paper Substrate for Paper-Spray Analysis of Therapeutic Drugs in Dried Blood Spots. Anal. Chem. 2012,84, 931-938.

8. Jhang C-S, Lee H, He Y-S, Liu J-T, Lin C-H. Rapid screening and determination of 4chloroamphetamine in saliva by paper spray-mass spectrometry and capillary electrophoresis-mass spectrometry. Electrophoresis 2012,33, 3073-3078.

9. Zhang ZP, Xu W, Manicke NE, Cooks RG, Ouyang Z. Silica Coated Paper Substrate for Paper-Spray Analysis of Therapeutic Drugs in Dried Blood Spots. Analytical Chemistry 2012,84, 931-938.

10. Su Y, Wang H, Liu J, Wei P, Cooks RG, Ouyang Z. Quantitative paper spray mass spectrometry analysis of drugs of abuse. Analyst 2013,138, 4443-4447.

11. Naccarato A, Moretti S, Sindona G, Tagarelli A. Identification and assay of underivatized urinary acylcarnitines by paper spray tandem mass spectrometry. Analytical and Bioanalytical Chemistry 2013,405, 8267-8276.

12. Wang H, Ren Y, Mcluckey MN et al. Direct Quantitative Analysis of Nicotine Alkaloids from Biofluid Samples using Paper Spray Mass Spectrometry. Anal. Chem. 2013,85, $11540-11544$.

13. Espy RD, Teunissen SF, Manicke NE et al. Paper Spray and Extraction Spray Mass Spectrometry for the Direct and Simultaneous Quantification of Eight Drugs of Abuse in Whole Blood. Anal. Chem. 2014,86, 7712-7718.

14. Shi R-Z, El Gierari ETM, Manicke NE, Faix JD. Rapid measurement of tacrolimus in whole blood by paper spray-tandem mass spectrometry (PS-MS/MS). Clinica Chimica Acta 2015,441, 99-104.

15. Shi R-Z, El Gierari ETM, Faix JD, Manicke NE. Rapid Measurement of Cyclosporine and Sirolimus in Whole Blood by Paper Spray-Tandem Mass Spectrometry. Clinical Chemistry In press, 
16. Soparawalla S, Tadjimukhamedov FK, Wiley JS, Ouyang Z, Cooks RG. In situ analysis of agrochemical residues on fruit using ambient ionization on a handheld mass spectrometer. Analyst 2011,136, 4392-4396.

17. Zhang Z, Cooks RG, Ouyang Z. Paper spray: a simple and efficient means of analysis of different contaminants in foodstuffs. Analyst 2012,137, 2556-2558.

18. Taverna D, Di Donna L, Mazzotti F, Policicchio B, Sindona G. High-throughput determination of Sudan Azo-dyes within powdered chili pepper by paper spray mass spectrometry. Journal of Mass Spectrometry 2013,48, 544-547.

19. Wang H, Manicke NE, Yang Q et al. Direct Analysis of Biological Tissue by Paper Spray Mass Spectrometry. Anal. Chem. 2011,83, 1197-1201.

20. Jain S, Heiser A, Venter AR. Spray desorption collection: an alternative to swabbing for pharmaceutical cleaning validation. Analyst 2011,136, 1298-1301.

21. Pirro V, Jarmusch AK, Vincenti M, Cooks RG. Direct drug analysis from oral fluid using medical swab touch spray mass spectrometry. Analytica Chimica Acta 2015,861, 47-54.

22. Oradu SA, Cooks RG. Multistep Mass Spectrometry Methodology for Direct Characterization of Polar Lipids in Green Microalgae using Paper Spray Ionization. Anal. Chem. 2012,84, 10576-10585.

23. Hamid AM, Jarmusch AK, Pirro V et al. Rapid Discrimination of Bacteria by Paper Spray Mass Spectrometry. Anal. Chem. 2014,86, 7500-7507.

24. Hamid AM, Wei P, Jarmusch AK, Pirro V, Cooks RG. Discrimination of Candida species by paper spray mass spectrometry. International Journal of Mass Spectrometry 2015,378, 288-293.

25. Liu W, Wang N, Lin X, Ma Y, Lin J-M. Interfacing Microsampling Droplets and Mass Spectrometry by Paper Spray Ionization for Online Chemical Monitoring of Cell Culture. Anal. Chem. 2014,86, 7128-7134.

26. Reeber SL, Gadi S, Huang S-B, Glish GL. Direct analysis of herbicides by paper spray ionization mass spectrometry. Analytical Methods 2015, doi:10.1039/C5AY02125A

27. Manicke NE, Belford M. Separation of Opiate Isomers Using Electrospray Ionization and Paper Spray Coupled to High-Field Asymmetric Waveform Ion Mobility Spectrometry. Journal of the American Society for Mass Spectrometry 2015,26, 701-705.

28. Zhang C, Manicke NE. Development of a Paper Spray Mass Spectrometry Cartridge with Integrated Solid Phase Extraction for Bioanalysis. Anal. Chem. 2015,87, 6212-6219.

29. Narayanan R, Sarkar D, Cooks RG, Pradeep T. Molecular Ionization from Carbon Nanotube Paper. Angew Chem Int Edit 2014,53, 5936-5940.

30. $\mathrm{Hu} \mathrm{B}$, So PK, Chen HW, Yao ZP. Electrospray Ionization Using Wooden Tips. Analytical Chemistry 2011,83, 8201-8207.

31. Zhang $\mathrm{M}$, Lin $\mathrm{FK}, \mathrm{Xu} \mathrm{JG}, \mathrm{Xu}$ W. Membrane Electrospray Ionization for Direct Ultrasensitive Biomarker Quantitation in Biofluids Using Mass Spectrometry. Analytical Chemistry 2015,87, 3123-3128.

32. Zhang Y, Li H, Ma Y, Lin J-M. Paper spray mass spectrometry-based method for analysis of droplets in a gravity-driven microfluidic chip. Analyst 2014,139, 1023-1029.

33. Liu W, Chen Q, Lin X, Lin J-M. Online multi-channel microfluidic chip-mass spectrometry and its application for quantifying noncovalent protein-protein interactions. Analyst 2015,140, 1551-1554. 
34. Gómez-Ríos GA, Pawliszyn J. Development of Coated Blade Spray Ionization Mass Spectrometry for the Quantitation of Target Analytes Present in Complex Matrices. Angewandte Chemie International Edition 2014,53, 14503-14507.

35. Deng JW, Yang YY, Fang L, Lin L, Zhou HY, Luan TG. Coupling Solid-Phase Microextraction with Ambient Mass Spectrometry Using Surface Coated Wooden-Tip Probe for Rapid Analysis of Ultra Trace Perfluorinated Compounds in Complex Samples. Analytical Chemistry 2014,86, 11159-11166.

36. Ren Y, Wang H, Liu J, Zhang Z, Mcluckey MN, Ouyang Z. Analysis of Biological Samples Using Paper Spray Mass Spectrometry: An Investigation of Impacts by the Substrates, Solvents and Elution Methods. Chromatographia 2013,76, 1339-1346.

37. Hu B, So P-K, Yao Z-P. Analytical Properties of Solid-substrate Electrospray Ionization Mass Spectrometry. Journal of the American Society for Mass Spectrometry 2013,24, 5765.

38. Oss M, Kruve A, Herodes K, Leito I. Electrospray Ionization Efficiency Scale of Organic Compounds. Analytical Chemistry 2010,82, 2865-2872.

39. Espy RD, Muliadi AR, Ouyang Z, Cooks RG. Spray mechanism in paper spray ionization. International Journal of Mass Spectrometry 2012,325, 167-171. 